\title{
Clinical Characteristics and Adequate Treatment of Familial Adenomatous Polyposis Combined with Desmoid Tumors
}

\section{Won Beom Jung, MD \\ Chan Wook Kim, MD \\ Jin Cheon Kim, MD}

Department of Surgery,

Institute of Innovative Cancer Research, Asan Medical Center,

University of Ulsan College of Medicine, Seoul, Korea

\section{Purpose}

The objective of this study was to examine the clincopathologic characteristics and outcomes of familial adenomatous polyposis (FAP) patients with and without desmoid tumors (DTs), including the risk factors for progression of FAP-related DTs.

\section{Materials and Methods}

We reviewed the medical records and database of all patients with FAP who were treated between January 1993 and December 2011.

\section{Results}

Of 75 FAP patients, 18 (24\%) were FAP with DTs. Seventeen of these had intra-abdominal DTs and one had intra- and extra-abdominal DTs. We divided the patients into two groups according to type of resection; the R0 or R1 resection group, referred to as the curative resection group (eight patients), and the $\mathrm{R} 2$ resection/palliative operation/medical treatment group, referred to as the palliative resection group (10 patients). Two patients in the curative resection group and two patients in the palliative group had progressed to tumor growth by the follow-up $(p=0.800)$. In univariate analysis, DT diagnosis before or simultaneously with FAP diagnosis (DTs unrelated to surgical trauma) was a significant risk factor for tumor progression at final follow-up.

\section{Conclusion}

A multidisciplinary approach to DT treatment is needed, including nonsteroidal antiinflammatory drugs, anti-estrogens, cytotoxic agents, and surgery. However, the role of surgery in resectable and complicated tumors may be limited. DT unrelated to surgical trauma has a relatively poor prognosis.
: 82-2-3010-3489

Fax: 82-2-474-9027

E-mail: jckim@amc.seoul.kr

Received October 1, 2013

Accepted November 8, 2013

*Won Beom Jung and Chan Wook Kim contributed equally to this work.
Key words

Familial adenomatous polyposis,

Aggressive fibromatosis, Risk factors

\section{Introduction}

Familial adenomatous polyposis (FAP) is a hereditary condition transmitted in an autosomal dominant fashion. It is characterized by development of hundreds to thousands of adenomatous polyps in the colon and rectum, leading to a nearly $100 \%$ risk of cancer by the age of 40 [1]. The condition results from mutation of the APC gene on chromosome 5 , and accounts for $1 \%$ or less of all colorectal cancers (CRCs)
[2]. The risk of death due to CRC is decreased after prophylactic total proctocolectomy. Therefore, the extra-colonic manifestations of FAP are becoming more clinically significant [3]. Among these, desmoid tumors (DTs) and periampullary cancers are currently the most common causes of death after CRC [4].

DTs are poorly understood, frequently aggressive, tumors of mesenchymal origin, which arise in musculoaponeurotic structures. Intra-abdominal desmoids often appear to be surgically-induced, typically by prophylactic colectomy, but 
can arise in the absence of surgery [5]. DTs are rare soft tissue tumors which can arise sporadically or in association with FAP. The DT is an extra-colonic manifestation of FAP. Despite their inability to metastasize, DTs are frequently locally invasive and may compress surrounding structures. In contrast to sporadic tumors, FAP-associated desmoids usually arise in the abdomen, and are a major cause of morbidity and mortality in patients who have undergone prophylactic colonic surgery. Despite recent research, they remain poorly understood entities, and current treatments generally lack evidence of efficacy.

In the current study, we retrospectively examined the clincopathologic characteristics and outcomes of FAP patients with and without DTs, as well as the risk factors for progression of FAP-related DTs in patients undergoing surgical and pharmacological therapies in a single center.

\section{Materials and Methods}

\section{Patients}

We reviewed the medical records and database of all patients with FAP treated between January 1993 and December 2011 at Asan Medical Center, Seoul, Korea. The diagnosis of DT was established by a combination of clinical examination, surgical exploration, and radiologic evaluation. A total of 75 FAP patients were evaluated.

For each patient, the status of desmoid growth at the final follow-up was assessed and categorized according to either 'regression or stabilization of tumor growth' or 'progression of tumor growth.'

Table 1. Clinical characteristics of FAP with and without desmoids

\begin{tabular}{|c|c|c|c|}
\hline & FAP with desmoids $(n=18)$ & FAP without desmoids $(n=57)$ & p-value \\
\hline Gender & & & 0.912 \\
\hline Male & $11(61.1)$ & $37(59.6)$ & \\
\hline Female & $7(38.9)$ & $23(40.4)$ & \\
\hline Age at diagnosed FAP (yr) & $28(19-45)$ & $30(13-78)$ & 0.264 \\
\hline Incidence of CRC & $5(27.8)$ & $20(35.1)$ & 0.566 \\
\hline AJCC stage of CRC & & & 0.018 \\
\hline 0/I/II/III & $3 / 0 / 0 / 2$ & $1 / 5 / 5 / 9$ & \\
\hline Pregnancy history & $3 / 7(42.8)$ & $12 / 23(52.1)$ & 0.907 \\
\hline \multicolumn{4}{|l|}{ Extracolonic manifestation } \\
\hline Osteoma & $2(11.1)$ & $5(8.8)$ & 0.766 \\
\hline Dental anomaly & $1(5.6)$ & $3(5.3)$ & 0.962 \\
\hline CHRPE & $1(5.6)$ & $8(14.0)$ & 0.334 \\
\hline Epidermal cyst & $5(27.8)$ & $1(1.8)$ & $<0.001$ \\
\hline Fundic gland polyps & $10(55.6)$ & $38(66.7)$ & 0.392 \\
\hline Papillary thyroid cancer & $3(16.7)$ & $3(5.3)$ & 0.120 \\
\hline Duodenum/small bowel adenoma & $4(22.2)$ & $27(47.4)$ & 0.059 \\
\hline Stomach cancer & $1(5.6)$ & $1(1.8)$ & 0.383 \\
\hline Adrenal adenoma & $2(11.1)$ & $3(5.3)$ & 0.386 \\
\hline Family history (CRC or FAP) & $11(61.1)$ & $21(36.8)$ & 0.070 \\
\hline Survival rate at final follow-up & $15(83.3)$ & $50(87.7)$ & 0.633 \\
\hline Cause of death & & & 0.007 \\
\hline CRC related & 0 & 4 & \\
\hline Desmoid related & 3 & 0 & \\
\hline Other causes & 0 & 3 & \\
\hline Follow-up (mo) & $76(19-203)$ & $59(2-247)$ & - \\
\hline
\end{tabular}

Values are presented as number (\%) or median (range). FAP, familial adenomatous polyposis; CRC, colorectal cancer; AJCC, American Joint Committee on Cancer; CHRPE, congenital hypertrophy of the retinal pigment epithelium. 
Table 2. Characteristics of FAP with desmoids

\begin{tabular}{lc} 
& FAP with desmoids (n=18) \\
Desmoid location & $12(66.7)$ \\
Mesentery & $3(16.7)$ \\
Abdominal wall & $1(5.5)$ \\
Mesentery+abdominal wall & $1(5.5)$ \\
Mesentery+abdominalwall+extra-abdominal & $1(5.5)$ \\
Anastomosis site & $7(2-23)$ \\
Size of first DT (cm) & $3(27.3)$ \\
Multiple desmoids & $3(42.9)$ \\
Male & $22(8-45)$ \\
Female & $3(16.7)$ \\
Time from FAP surgery to DT diagnosis (mo) & \\
DT diagnosed before FAP diagnosis or at the same time & \\
\hline
\end{tabular}

Values are presented as number (\%) or median (range). FAP, familial adenomatous polyposis; DT, desmoid tumor.

\section{Statistical analysis}

Categorical variables were compared using chi-squared tests, and continuous variables were compared using independent sample t-tests. The Kaplan-Meier method was used for comparison of overall survival rates. All statistical tests were two-sided, and $\mathrm{p}<0.05$ was considered statistically significant. Statistical analyses were performed using SPSS ver. 19.0 for Windows (SPSS, Inc., Chicago, IL).

\section{Results}

\section{Characteristics of FAP patients with and without desmoids}

Of the 75 patients, $18(24 \%)$ were FAP with DTs. The characteristics of FAP patients with and without DTs are summarized in Table 1. The FAP with and without DT groups showed similar incidence of CRC (5 [27.8\%] vs. 20 [35.1\%], $\mathrm{p}=0.566)$. However, advanced stage CRC was more frequent in the FAP without DT group. Therefore, the main cause of deaths due to FAP without DTs was CRC-related $(4 / 7,57.1 \%)$, while deaths due to FAP with DTs were mainly desmoid-related. The two groups showed similar 5-year overall survival rates $(88.9 \%$ in the FAP with DT group vs. $90.1 \%$ in the FAP without DT group, log rank=0.846) (Fig. 1).

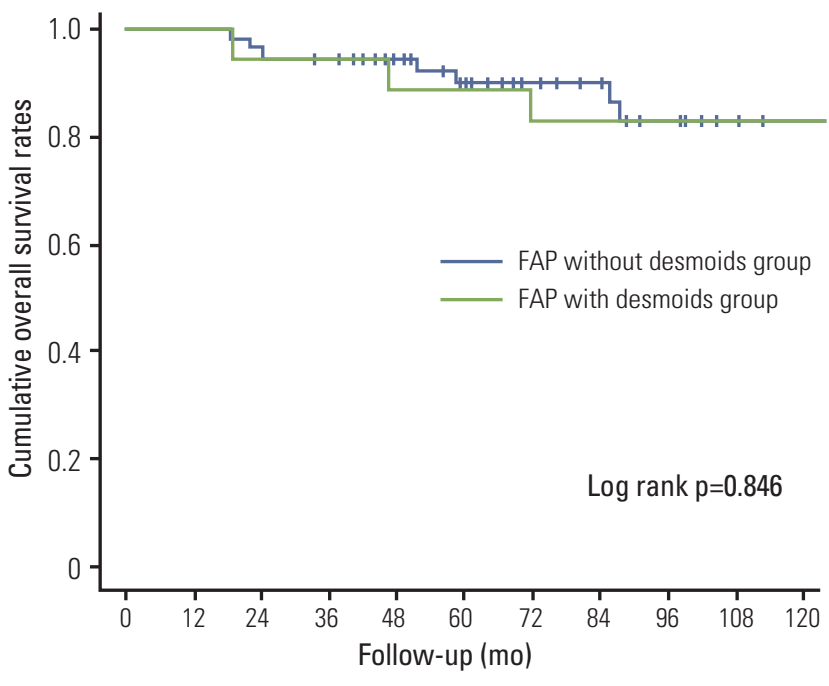

Fig. 1. Overall survival curves of familial adenomatous polyposis (FAP) with and without desmoid tumors.

\section{Clinicopathologic characteristics of DT}

Seventeen of the 18 patients with DTs had intra-abdominal DTs and one had intra- and extra-abdominal DTs. The median age at time of diagnosis of DTs was 30 years (range, 20 to 48 years). Six of the 18 patients had multiple DTs. Two of the 18 patients were diagnosed with DTs before FAP diagnosis, and one was diagnosed with DT and FAP simultaneously. The median time between colectomy and DT 
Table 3. Surgical outcomes according to resection type

$\begin{array}{lccc} & \text { Curative resection group (n=8) } & \text { Palliative resection group (n=10) } & \text { p-value } \\ \text { Status at final follow-up } & 6(75.0) & 9(90.0) & 0.396 \\ \text { Alive } & 2(25.0) & 1(10.0) & \\ \text { Dead due to DT } & 6(75.0) & 8(80.0) & 0.800 \\ \text { DT status at final follow-up } & 2(25.0) & 2(20.0) & \\ \text { Regression/stable } & & \\ \text { Progression/variable } & \end{array}$

Values are presented as number (\%). DT, desmoid tumor.

Table 4. Treatment outcomes of patients with desmoid tumors who underwent palliative surgery

\begin{tabular}{|c|c|c|c|c|c|}
\hline Gender & Site DT & Age (yr) & Treatment & $\begin{array}{l}\text { Effect on } \\
\text { desmoid growth }\end{array}$ & $\begin{array}{l}\text { Follow-up } \\
\qquad(\mathrm{mo})\end{array}$ \\
\hline \multirow[t]{7}{*}{ Female } & \multirow{7}{*}{$\begin{array}{l}\text { Intra- and extra- } \\
\text { abdominal DT }\end{array}$} & \multirow[t]{7}{*}{24} & Sulindac, tamoxifen & Progression & 7 \\
\hline & & & Sutene & Progression and tumor perforation & 6 \\
\hline & & & R2 DT resection and palliative ICR & - & 1 \\
\hline & & & Adriamycin + dacarbazin + meloxicam & Progression & 24 \\
\hline & & & $\mathrm{R} 2 \mathrm{DT}$ resection & - & \\
\hline & & & Doxorubicin + dacarbazine + meloxicam & Progression & 7 \\
\hline & & & RTx & Progression & 4 \\
\hline \multirow[t]{3}{*}{ Male } & \multirow[t]{3}{*}{ Mesentery } & 45 & TPC IPAA & - & 15 \\
\hline & & 47 & Unresectable DT, O\&C & - & 0 \\
\hline & & & Tamoxifen + sulindac & Stabilization & 56 \\
\hline \multirow[t]{3}{*}{ Male } & \multirow[t]{3}{*}{ Mesentery } & 34 & TPC IPAA & - & 57 \\
\hline & & 38 & Unresectable DT, open biopsy & - & 0 \\
\hline & & & Tamoxifen, sulindac & Stabilization & 46 \\
\hline \multirow[t]{4}{*}{ Male } & \multirow[t]{4}{*}{ Mesentery } & 33 & TPC IPAA & - & 23 \\
\hline & & 35 & Unresectable DT, O\&C & - & 0 \\
\hline & & & Tamoxifen+LHRH agonist+Glivec & Stabilization & 4 \\
\hline & & & Tamoxifen+LHRH agonist & Stabilization & 84 \\
\hline \multirow[t]{4}{*}{ Female } & \multirow[t]{4}{*}{ Mesentery } & 45 & TPC IPAA & - & 36 \\
\hline & & 48 & Unresectable DT, palliative ileostomy & Progression & 9 \\
\hline & & & Glivec & Progression & 2 \\
\hline & & & Sutene & Stabilization & 72 \\
\hline \multirow[t]{3}{*}{ Male } & \multirow[t]{3}{*}{ Mesentery } & 36 & TPC IPAA & - & 10 \\
\hline & & 37 & Unresectable DT, bypass and ileostomy & Progression & 4 \\
\hline & & & Tamoxifen+sulindac & Progression, death & 7 \\
\hline
\end{tabular}

DT, desmoid tumor; ICR, ileocecal resection; RTx, radiotherapy; TPC IPAA, total proctocolectomy with ileal pouch anal anastomosis; O\&C, open and closure; LHRH, luteinizing hormone releasing hormone.

diagnosis in the remaining 15 patients was 22 months (range, 8 to 45 months). The characteristics of the DTs are summarized in Table 2.

\section{Outcomes of DTs according to resection type}

Six patients underwent complete resection (R0), two patients $\mathrm{R} 1$ resection and six patients palliative resection (one patient, R2 resection; three patients, open biopsy; two 
patients, ileostomy). The reasons for palliative surgery were abdominal discomfort due to the effect of the mass in five patients, and desmoid perforation in the one remaining patient.

The four remaining patients received medical treatment for resectable DT. Two of them received non-cytotoxic pharmacological therapy, one received both non-cytotoxic and cytotoxic therapy, and one received conservative treatment (wait-and-see).

We divided the patients into two groups according to resection type; an $\mathrm{R} 0$ or $\mathrm{R} 1$ resection group, categorized as the curative resection group, and an $\mathrm{R} 2$ resection, palliative operation, or medical treatment group, categorized as the palliative resection group. Two of the eight patients in the curative resection group and two of the ten patients in the palliative group had progressed to tumor growth by the last follow-up ( $p=0.800$ ). Two patients in the curative resection group and one patient in the palliative resection group died as a result of DTs $(\mathrm{p}=0.936)$ (Table 3$)$.

\section{Long-term outcomes and risk factors for progression of disease}

Five of eight patients with desmoids deemed to have been completely excised had recurrence of DTs. Two of these five patients underwent further resection and pharmacotherapy, two were treated with salvage chemotherapy after failed non-cytotoxic pharmacotherapy, and one was treated with non-cytotoxic pharmacotherapy alone. The median interval for recurrence of disease after surgical intervention was 15 months (range, 13 to 25 months). The two patients who underwent further resection of their recurrent disease died as a result of disease progression and bowel perforation despite pharmacotherapy and radiation treatment.

Four of the six patients who underwent palliative surgery were treated with nonsteroidal anti-inflammatory drugs (NSAIDs) and hormonal agents after surgery. DTs were stabilized after treatment in three patients. In one patient treated with imatinib and sunitinib, the disease was stabilized after treatment with sunitinib. The other patient was treated with antisarcomatic agents; however, the disease progressed in spite of treatment (Table 4).

The patient who underwent ileostomy because of unresectable DT died as a result of disease progression. In univariate analysis, DT diagnosis before or concurrent with FAP diagnosis (DTs unrelated to surgical trauma) was a significant risk factor for tumor progression by the end of follow-up (Table 5).

\section{Discussion}

DTs are rare, with an estimated incidence of 2-5 per million population. The prevalence of DTs in FAP patients is probably between $10 \%$ and $15 \%$, approximately 1,000 times that in the general population [6,7]. However, the etiology, behavior, and tumor biology of DT in patients with FAP remain poorly understood. Surgical trauma, pregnancy, and other hormonal influences have been implicated in the development of DT [6,8]. In addition, presence of an APC mutation at the $3^{\prime}$ end of the APC gene is more likely to result in development of DT $[9,10]$. Some studies $[8,11]$ reported that multiple desmoids are more likely to occur in women. However, in this study, there was no difference in the proportion of multiple desmoids by gender. In general, DT affects $10-15 \%$ of patients with FAP. However, in this study, the incidence was $24 \%$, greater than previously reported in the literature. Five out of 18 desmoid patients were referred from other hospitals after the FAP operation or recurrence of

Table 5. Univariate analysis of risk factors for tumor progression

\begin{tabular}{|c|c|c|c|}
\hline & Stabilization $(n=14)$ & Progression (n=4) & p-value \\
\hline Gender & & & 0.093 \\
\hline Male & $10(71.4)$ & $1(25.0)$ & \\
\hline Female & $4(28.6)$ & $3(75.0)$ & \\
\hline Age at DT diagnosis (yr) & $32(20-48)$ & $27(20-37)$ & 0.411 \\
\hline $\begin{array}{l}\text { Diagnosis of DT before FAP } \\
\text { diagnosis or simultaneously }\end{array}$ & 0 & $3(75.0)$ & $<0.001$ \\
\hline Survival rates at last follow-up & $14(100)$ & $1(25.0)$ & $<0.001$ \\
\hline Incidence of CRC & $5(35.7)$ & 0 & 0.160 \\
\hline Curative resection & $6(42.9)$ & $2(50.0)$ & 0.800 \\
\hline
\end{tabular}

Values are presented as number (\%) or median (range). DT, desmoid tumor; FAP, familial adenomatous polyposis; CRC, colorectal cancer. 
DTs. Therefore, the relatively greater incidence of DT observed in our study may be due to the reputation of our center as a higher referral center in Korea.

Some studies have compared sporadic and FAP-related desmoids tumors $[12,13]$. Patients with FAP related DTs were younger at the time of diagnosis and had CRC more often than patients with sporadic DTs. The proportion of female patients with sporadic DTs was higher than that of males, and half of sporadic DTs were located extra-abdominally, whereas FAP-related DTs were generally intra-abdominal.

The optimal treatment for DTs in FAP patients has not been established; medical and surgical treatments have not been standardized, owing to a lack of understanding of the tumor biology, the rarity of the disease, and the lack of predictably effective agents.

Previous studies reported high recurrence rates and high morbidity rates after surgical resection $[7,14]$. On the other hand, surgery for intra-abdominal desmoids as well as for abdominal wall and extra-abdominal tumors has been shown to be safe in selected patients [11]. Comparable outcomes were recently reported for surgery and conservative treatment for intra-abdominal desmoids [15]. Surgical treatment is generally more suitable for extra-abdominal and abdominal wall DTs than for mesenteric desmoids $[8,11,15]$.

Commonly used pharmacological agents are NSAIDs and hormonal agents. One systematic review reported favorable outcomes after using NSAIDs, hormonal agents, and cytotoxic chemotherapies [16]. Another study suggested that high-dose tamoxifen $(120 \mathrm{mg}$ ) and sulindac $(300 \mathrm{mg})$ were the primary treatment for patients with FAP-associated DTs [17]. A recent study found no significant difference in progression-free survival between NSAIDs, hormonal treatment, and a combination of the two [15]. In the current study, four of the six patients who underwent palliative surgery were treated with NSAIDs and hormonal treatment after surgery, and the DTs were stabilized in three patients. Hormonal treatment with NSAIDs also appeared to have a positive effect on unresectable DTs.

Preliminary results for use of imatinib mesylate in treatment of DTs have been encouraging, with partial response rates of $10-15 \%$, and one-year tumor control rates of $36-50 \%$ [18,19]. In another study imatinib had no evident positive effect [15]. In our study, in two of the three patients treated with imatinib, this treatment was effective against DTs. Doxorubicin based chemotherapy has recently been reported to be an effective and safe treatment for FAPassociated DTs [15,20-22]. In our study, the DTs were stabilized in one of two patients treated with doxorubicinbased chemotherapy.

There is increasing evidence supporting the use of radiotherapy either before surgery or in combination with surgery, to reduce local recurrence of abdominal wall and extra-abdominal desmoids $[23,24]$. However, radiotherapy is rarely used for intra-abdominal desmoids because of the risk of radiation enteritis.

A recent study proposed a staging system based on tumor size, symptoms, behavior (growth), and the presence of complications, and the authors tailored their treatment of intra-abdominal DTs according to the stage. Stage I desmoids were watched carefully, and the patients were only occasionally started on NSAID. If a stage I desmoid was found incidentally during surgery and was easily resectable, resection was performed. Stage II tumors were treated by surgery (if resectable with minimal sequelae), and anti-estrogens or NSAIDs were also used whenever possible in order to obtain a faster and more consistent response with a low risk of adverse effects. Stage III desmoids were treated with a combination of NSAID, anti-estrogens, and cytotoxic agents, and patients with stage IV disease received urgent treatment, including major exenterative surgery whenever possible, as well as cytotoxic agents and radiation [25].

In our study, no difference in mortality and progression rate was observed between the curative resection group and the palliative resection group. Intra-abdominal DTs were a sign of locally aggressive tumors with unpredictable behavior requiring multidisciplinary treatment. Due to the possibility of massive small bowel resection, we recommend medical treatment in cases of mesentery DTs. In cases of abdominal wall DTs, we recommend surgical resection. Surgical intervention was also required in cases of bowel obstruction, fistula, and tumor rupture. We believe that the role of surgery is limited to resectable tumors and tumors with complications such as fistulae and bowel obstructions. Although there was no established strategy for medical therapy of DT in our center, we usually started the treatment with sulindac and tamoxifen or tamoxifen and goserelin acetate. We consider treatment with a cytotoxic agent such as doxorubicin-based chemotherapy or imatinib mesylate after failure of primary treatment.

Surgical trauma is a well-known risk factor for desmoids in patients with FAP. Some authors recommend delayed prophylactic surgery for female patients with $3^{\prime} A P C$ mutations, since development of DTs is quite likely in these patients $[3,24]$. In the current study, we found that patients with DTs that were not the consequence of surgical trauma were at increased risk of tumor progression and this showed an association with tumor-related death. DTs unrelated to surgical trauma appeared to relapse despite curative resection. However, this was a small study and a large-scale multicenter study is needed in order to confirm our conclusions. 


\section{Conclusion}

DTs in patients with FAP are mainly intra-abdominal, particularly mesenteric. A multidisciplinary approach to DT treatment that includes NSAIDs, anti-estrogens, cytotoxic agents, and surgery is needed. However, the role of surgery may be limited to resectable tumors and tumors with complications. Patients with DTs unrelated to surgical trauma appear to have a relatively poor prognosis.

\section{Acknowledgments}

This study was supported by grants (to J.C. Kim) from the Asan Institute for Life Sciences (2013-069), the Korea Health 21 R\&D Project (A062254), and the Center for Development and Commercialization of Anti-Cancer Therapeutics (A102059), Ministry of Health, Welfare, and Family Affairs, Republic of Korea.

\section{Conflicts of Interest}

Conflict of interest relevant to this article was not reported.

\section{References}

1. Lynch HT, Lynch JF, Lynch PM, Attard T. Hereditary colorectal cancer syndromes: molecular genetics, genetic counseling, diagnosis and management. Fam Cancer. 2008;7:27-39.

2. Vasen HF, Moslein G, Alonso A, Aretz S, Bernstein I, Bertario $\mathrm{L}$, et al. Guidelines for the clinical management of familial adenomatous polyposis (FAP). Gut. 2008;57:704-13.

3. Sinha A, Tekkis PP, Gibbons DC, Phillips RK, Clark SK. Risk factors predicting desmoid occurrence in patients with familial adenomatous polyposis: a meta-analysis. Colorectal Dis. 2011;13:1222-9.

4. Arvanitis ML, Jagelman DG, Fazio VW, Lavery IC, McGannon E. Mortality in patients with familial adenomatous polyposis. Dis Colon Rectum. 1990;33:639-42.

5. Sturt NJ, Clark SK. Current ideas in desmoid tumours. Fam Cancer. 2006;5:275-85.

6. Gurbuz AK, Giardiello FM, Petersen GM, Krush AJ, Offerhaus GJ, Booker SV, et al. Desmoid tumours in familial adenomatous polyposis. Gut. 1994;35:377-81.

7. Rodriguez-Bigas MA, Mahoney MC, Karakousis CP, Petrelli NJ. Desmoid tumors in patients with familial adenomatous polyposis. Cancer. 1994;74:1270-4.

8. Clark SK, Neale KF, Landgrebe JC, Phillips RK. Desmoid tumours complicating familial adenomatous polyposis. Br J Surg. 1999;86:1185-9.

9. Speake D, Evans DG, Lalloo F, Scott NA, Hill J. Desmoid tumours in patients with familial adenomatous polyposis and desmoid region adenomatous polyposis coli mutations. Br J Surg. 2007;94:1009-13.

10. Scott RJ, Froggatt NJ, Trembath RC, Evans DG, Hodgson SV, Maher ER. Familial infiltrative fibromatosis (desmoid tumours) (MIM135290) caused by a recurrent 3' APC gene mutation. Hum Mol Genet. 1996;5:1921-4.
11. Latchford AR, Sturt NJ, Neale K, Rogers PA, Phillips RK. A 10-year review of surgery for desmoid disease associated with familial adenomatous polyposis. Br J Surg. 2006;93:1258-64.

12. Nieuwenhuis MH, Casparie M, Mathus-Vliegen LM, Dekkers OM, Hogendoorn PC, Vasen HF. A nation-wide study comparing sporadic and familial adenomatous polyposisrelated desmoid-type fibromatoses. Int J Cancer. 2011;129: 256-61.

13. Kim DD, Yu CS, Hong DH, Jung SH, Choi PW, Park IJ, et al. Desmoid tumor in familial adenomatous polyposis (FAP). J Korean Soc Coloproctol. 2008;24:20-6.

14. Heiskanen I, Jarvinen HJ. Occurrence of desmoid tumours in familial adenomatous polyposis and results of treatment. Int J Colorectal Dis. 1996;11:157-62.

15. Nieuwenhuis MH, Mathus-Vliegen EM, Baeten CG, Nagengast FM, van der Bijl J, van Dalsen AD, et al. Evaluation of management of desmoid tumours associated with familial adenomatous polyposis in Dutch patients. Br J Cancer. 2011;104:37-42.

16. Janinis J, Patriki M, Vini L, Aravantinos G, Whelan JS. The pharmacological treatment of aggressive fibromatosis: a systematic review. Ann Oncol. 2003;14:181-90.

17. Hansmann A, Adolph C, Vogel T, Unger A, Moeslein G. High-dose tamoxifen and sulindac as first-line treatment for desmoid tumors. Cancer. 2004;100:612-20.

18. Heinrich MC, Joensuu H, Demetri GD, Corless CL, Apperley J, Fletcher JA, et al. Phase II, open-label study evaluating the activity of imatinib in treating life-threatening malignancies known to be associated with imatinib-sensitive tyrosine kinases. Clin Cancer Res. 2008;14:2717-25.

19. Heinrich MC, McArthur GA, Demetri GD, Joensuu H, Bono $\mathrm{P}$, Herrmann R, et al. Clinical and molecular studies of the 
effect of imatinib on advanced aggressive fibromatosis (desmoid tumor). J Clin Oncol. 2006;24:1195-203.

20. Gega M, Yanagi H, Yoshikawa R, Noda M, Ikeuchi H, Tsukamoto K, et al. Successful chemotherapeutic modality of doxorubicin plus dacarbazine for the treatment of desmoid tumors in association with familial adenomatous polyposis. J Clin Oncol. 2006;24:102-5.

21. Constantinidou A, Jones RL, Scurr M, Al-Muderis O, Judson I. Pegylated liposomal doxorubicin, an effective, well-tolerated treatment for refractory aggressive fibromatosis. Eur J Cancer. 2009;45:2930-4.

22. Constantinidou A, Jones RL, Scurr M, Al-Muderis O, Judson
I. Advanced aggressive fibromatosis: Effective palliation with chemotherapy. Acta Oncol. 2011;50:455-61.

23. O'Dea FJ, Wunder J, Bell RS, Griffin AM, Catton C, O'Sullivan B. Preoperative radiotherapy is effective in the treatment of fibromatosis. Clin Orthop Relat Res. 2003;(415):19-24.

24. Durno C, Monga N, Bapat B, Berk T, Cohen Z, Gallinger S. Does early colectomy increase desmoid risk in familial adenomatous polyposis? Clin Gastroenterol Hepatol. 2007;5:1190-4.

25. Quintini C, Ward G, Shatnawei A, Xhaja X, Hashimoto K, Steiger E, et al. Mortality of intra-abdominal desmoid tumors in patients with familial adenomatous polyposis: a single center review of 154 patients. Ann Surg. 2012;255:511-6. 